Letras, Lima 48 (84-85): 71-83, 1976.

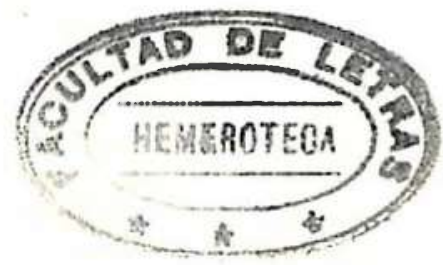

\title{
Modus Ponens e inducción matemática
}

\author{
LUIS PISCOYA HERMOZA
}

\begin{abstract}
Ciertamente un argumento de hecho para justificar una reflexión sobre el Modus Ponens (al que en adelante nos referiremos usando la abreviación MP), puede estar dado por la constatación de que los sistemas lógicos más conocidos (1) lo utilizan como única regla primitiva de inferencia. Asimismo, evidentemente, la solvencia de los sistemas que lo usan no se reduce al mero hecho de ser conocidos sino que han devenido en tales en la medida que satisfacen de manera lógicamente demostrada importantes propiedades metateóricas (2) y por esta razón se recurre a menudo a
\end{abstract}

(1) A fin de ser precisos indicamos que consideramos sistemas conocidos: el de Whitehead ye Russell,elel denHilbert y Bernays, el de Rosser, el de Lukasiewicz, etc. El hecho de que estos sistemas utilizan el MP como única regla primitiva de inferencia puede ser constatado en el caso de Whitehead y Russell revisando el primer volumen de Principia Mathematica (p. 94). En el caso del sistema Hilbert-Bernays puede recurrirse a la presentación hecha por Manuel Sacristán en Introducción a la lógica y al análisis formal (p. 110). La presentación de Sacristán ha sido tomada de Grundzäge der theoretischen Logik. Respecto al sistema de Rosser puede consultarse la clara presentación hecha por Copi en Symbolic Logic (p. 201). El sistema de Lukasiewicz que hemos aludido es el presentado por este autor en su obra Aristotle's Syllogistic (p. 80). En todos estos sistemas el MP es la única regla primitiva de inferencia en el sentido de que es la única que permite realizar la operación de separación (detachment), consistente en independizar el consecuente de un condicional de su respectivo antecedente, $y$ en el de que cualquier otra regla de inferencia se deriva en ellos como teorema por un número finito de aplicaciones del MP.

(2) Consideramos propiedades metateóricas de un sistema la consistencia, la compleción, la decidibilidad, etc. que algunos tratadistas prefieren denominar epiteóricas, como Haskell Curry que reserva la palabra 'metateoría' para referirse a un conjunto muy específico de cuestiones que se afrontan desde el punto de vista lingǘstico y filosófico, de modo que la metateoría queda incluida en la epiteoria. 
ellos para convalidar pruebas. Por citar sólo un ejemplo de este modo de proceder, el notable investigador Hao Wang, para probar que un sistema lógico $\mathrm{S}$ es completo, en su trabajo Some formal details on predicative set theories (3), prueba que $S$ es equivalente a un sistema $S^{\prime}$ del tipo Hilbert-Bernays cuya propiedad metateórica de compleción es conocida $y$, por este expediente, establece el teorema.

Sin embargo, no es el propósito del presente trabajo hacer un análisis del estatuto especial que tiene el MP como regla de inferencia en los sistemas lógicos ni defender tesis alguna acerca de lo que pueda considerarse su naturaleza misma. Lo que pretendemos fundamentalmente es examinar el tipo de relación que existe entre la mencionada regla de inferencia $y$ un principio conocido e importante como es el llamado Principio de Inducción Matemática o quinto postulado de Peano (4), que de una manera informal e intuitiva puede formularse en términos de: "si un teorema es válido para el número 1 y si se demuestra que es verdadero para $n+1$ siempre que lo es para $n$, será verdadero para todos los números enteros". Consecuentemente, el sentido de nuestro análisis está dirigido hacia los presupuestos lógicos del quehacer matemático.

Como es sabido el MP, dado que la noción convencional de prueba es de carácter finitístico, es insuficiente para establecer pruebas válidas para un conjunto infinito de objetos, las que sí pueden ser construidas por aplicación del Principio de Inducción Matemática. Empero, nosotros queremos enfatizar el hecho de que lo anterior no significa que el MP secencuentre ausente en tales pruebas o que éstas expresen un estilo de pensamiento radicalmente distinto al formulado a través del MP. Lo que nos proponemos probar con nuestra argumentación es que la presencia del MP en toda demostración por inducción matemática es inexcusable. Esto es, nuestra tesis es que la utilización del MP es condición necesaria para la aplicación legítima del Principio de Inducción Matemática (al que en adelante nos referiremos con la abreviación PIM) en las denominadas demostraciones inductivas $y$, consecuentemente, toda formalización de la aritmética y cualquier otro formalismo que contenga pruebas inductivas presuponen la validez del MP como elemento fundamental de su lógica subyacente.

(3) Este trabajo está contenido en el libro de Hao Wang titulado Logic, Computer and Sets, New York, Chelsea Publishing Company, 1970 , p. 585 .

(4) Según Polya, en Matemática y razonamiento plausible, el primero en conocer el método de demostración por inducción matemática fue Pascal. Esta información la ha tomado de las investigaciones de Freundenthal. 
Nuestra tesis la probaremos en tres partes. En la primera tras taremos lo relacionado con la función del MP en las pruebas por inducción matemática. En la segunda lo referente a la función del MP en las formalizaciones lógicas de la aritmética. Y en la parte final haremos una extensión, interesante fundamentalmente para los lectores no-matemáticos, de la tesis propuesta al llamado principio de inducción fuerte.

1. El matemático francés Henri Poincaré on su artículo titulado Naturaleza del razonamiento matemático (5), preocupado por establecer la validez de lo que él llamaba demostraciones por rocurrencia, señalaba que el PIM no era otra cosa que una proposición que condensa una infínidad de inferencias del tipo siguiente:

El teorema es cierto para el número 1

Luego, si es cierto para 1 , es cierto para 2

Entonces es cierto para 2

Luego, si es cierto para 2, es cierto para 3

Entonces es cierto para 3 ,

y así sucesivamente...

Poincoré denominó a esto una cadena de silogismos hipotéti. cos, sin embargo, como es claro, se trata mós bien de una cadena de aplicaciones de lo que los lógicos llaman universalmente regla del MP. El silogismo hipotético ordinario, que no es otra cosa que la expresión de la propiedad de transitividad para el condicional, a diferencia de lo que Poincutré pensaba, sólo nos autoriza $\alpha$ establecer que el primer antecedente implica el último consecuente de la cadena de condicionales pero no nos permite afirmar categóricamente que el teorema se cumple para los números 2 , $3, \ldots$ por separado, a menos que presupongamos la validez de alguna regla que autorice la separación del consecuente en las condiciones dadas.

En el planteamiento descrito parece que de alguna monera se está suponiendo la posibilidad de definir el cuantificador universal en los siguientes términos:

$$
\text { Df. 1. }(x) P(x) \equiv P a_{1}, P a_{2}, P a_{3} \text {. }
$$

En la definictón Df. debe asumirse, de conformidad con la idea de Poincaré, que el componente derecho de la equivalencia es un conjunto ífinito de elementos $\mathrm{Pa}_{1}$ cada uno de ellos obteni. do por MP. Pero para que la definición sea correcta no es sufi-

(5) Publicado en la selección de ensayos de Poincaré Filosofia de in clencia, México, UNAM, 1964, p. 217. 
ciente que cada proposición $\mathrm{Pa}_{1}$ sea verdadera sino que todas juntas constituyan una proposición verdadera, la misma que no podría ser otra cosa que una hipotética conjunción de infinitos componentes entendida como una función de verdad. Sin embargo, no es posible definir, al menos en términos convencionales, una función de verdad conjuntiva constituida por infinitos componentes debido a que no sería posible determinar en cada caso el valor de la pretendida conjunción infinitaria. No hay pues, en el sentido presupuesto por la tesis de Poincaré, conjunciones infinitas de valor de verdad calculable aunque, obviamente, se admite infinitas conjunciones finitas.

El matemático francés era consciente de la limitación antes señalada, pero en el ensayo citado no proporciona mayor detalle al respecto. La solución que propone es que la equivalencia establecida por Df. l es posible en el espíritu por una operación de síntesis muy especial que se impone a nuestra razón. De esta manera considera al PIM un ejemplo típico de juicio sintético a priori que expresa la naturaleza intrínseca del razonamiento matemático de manera especialmente privilegiada (6).

No es necesario identificarnos con el apriorismo de $\mathrm{H}$. Poincaré para reconocer el mérito que tiene por haber intuido en su tiempo una relación estrecha entre una regla tradicionalmente lógica como el MP y un principio tradicionalmente matemático como el PIM. Consideramos que su planteamiento es reelaborable y sostenible si se lo interpreta en el sentido que defendemos en nuestro trabajo, éste es que la validez del MP está presupuesta necesariamente en la construcción de pruebase por inducción matemática. Nuestra argumentación es como sigue

Si formalizamos el PIM de la manera usual, entonces tenemos:

$$
\text { "P(1) . }(\mathrm{x})(\mathrm{P}(\mathrm{x}) \rightarrow \mathrm{P}(\mathrm{x}+1)) \rightarrow(\mathrm{y}) \mathrm{P}(\mathrm{y}) "
$$

Claramente, en este caso para justificar la validez de la postulación del PIM ningún matemático incluiría como argumento el hecho de que un condicional con el antecedente falso es siempre verdadero como ocurre con algunas demostraciones del tipo de las usadas, por ejemplo, para probar que el conjunto vacío está contenido en todo conjunto, porque de hacerlo eliminaría la posibilidad de obtener una conclusión por separación del consecuente. Por tanto, toda demostración matemática por inducción asume la validez de la fórmula anterior y luego procede, de acuerdo a

(6) Es importante señalar que la concepción de Poincaré del PIM como juicio sintético a priori demuestra que es dudoso el convencionalismo radical que usualmente se le atribuye. 
la naturaleza del caso particular, a probar que las condiciones $P(1)$ y $(x)(P(x) \rightarrow P(x+1))$ son verdaderas. A continuactón deriva la validez del teorema para todos los números naturales mediante la denominada generalización inductiva que consiste en la afirmación de la fórmula $(y) P(y)$, lo cual sólo es posible legítimamente si se supone como dada una aplicación de la regla de Modus Ponens. Como paso intermedio, dentro de la demostración de la verdad de la condición $(x)(P(x) \rightarrow P(x+1))$, es necesaria una aplicación de la regla de generalización univer. sal para que el antecedente de la fórmula que expresa el PIM pueda coincidir exactamente con esta condición que los matemáticos usualmente la dejom indicada sin cuantificarla. Lo que hace posible la introducción del cuantificador universal es el empleo de la regla de la prueba condicional o del teorema de la deducción, según sea el caso. En la práctica matemática usual no se hace explícito el proceso lógico descrito, pero, en razón de la naturaleza de nuestra indagación, este análisis ha sido necesario para contar con las bases que hagan posible la reconstrucción de la estructura lógica de una prueba matemática por inducción de tal manera que queden explícitos sus presupuestos deductivos.

En armonía con lo anterior, consideramos que el siguiente es el esquema lógico de una prueba matemática basada en el PIM.

$$
\begin{aligned}
& \text { 1. } P(1) \cdot(x)(P(x) \rightarrow P(x+1)) \rightarrow(y) P(y) \\
& \text { 2. } P(1) \\
& \text { 3. }(x)(P(x) \rightarrow P(x+1)) \\
& \text { 4. } P(1) \cdot(x)(P(x) \rightarrow P(x+d)) \text { etras } \\
& \text { 5. }(y) P(y)
\end{aligned}
$$

Como puede apreciarse en el esquema de prueba anterior, la justificación de 5 sólo es posible por aplicación de alguna regla que autorice la separación del consecuente de la fórmula de la línea l. de su antecedente, y tal regla no puede ser otra que el MP aplicado a las líneas 1 . y 4 . De otra parte cualquier otra regla que se invocara sería equivalente al MP pues cumpliría la misma función $\mathrm{y}$, consecuentemente, no sería propicmente otra regla. Asimismo, si prescindiéramos de las líneas l. y 4. y del MP, como usualmente hacen los matemáticos, entonces no habría ninguna razón lógica explícita que nos cutorice a derivar la línea 5. desde las líneas 2. y 3., que por sí mismas son absolutamente insuficientes para justificarla. La derivación de 5 . así, desde el punto de vista formal, parecería gratuita. Y afirmamos que la derivación de 5. solamente desde 2. y 3. sería gratuita por el hecho de que existen estructuras matemáticas no inductivas que nos permiten construir interpretaciones que convierten en verdaderas a las premisas 2. y 3. y en falsa a la conclusión 5. Así, por ejemplo, 
para la interpretación que tiene como dominio el conjunto de los polinomios que tienen coeficiente principal no negativo y para los cuales se define las usuales operaciones de adición y multiplicación, se demuestra que en ella no es válido el PIM debido a que previamente se prueba que en esta estructura no es válida la propiedad de la división euclidiana. Por tanto, en este caso ocurre que 2. y 3. son proposiciones verdaderas y 5 . falsa, lo que impide que se pueda hablar de inferencia lógicamente válida que asegure a partir de la verdad de las premisas la verdad de la conclusión. Esto apoya nuestro argumento que sostiene que si se prescindiera de las líneas 1 . y 4., entonces la conclusión 5 . sería gratuita en el sentido de que no se seguiría con necesidad lógica (7). Empero si se toma el esquema de prueba completo, esto es, incluyendo desde la línea 1 . hasta la línea 5., entonces ocurre que la interpretación anterior ya no convierte en verdadera a la conjunción de las premisas (constituida por las cuatro primeras líneas) y en falsa a la conclusión 5., pues al ser falsa para tal interpretación la premisa l. (el PIM), la conjunción de las premisas ya no puede ser verdadera. De otra parte, podemos probar que para el esquema de demostración completo se cumple necesariamente que toda interpretación que convierte en verdadera a la conjunción de sus premisas también convierte en verdadera a la conclusión, esto es, para nuestro esquema se cumple la usual definición de consecuencia lógica. La condición anterior en efecto se cumple porque es evidente que si una interpretación convierte en verdadera a la conjunción de premisas, convierte también necesariamente pen verdaderas a l l y a 4. y, por consiguiente, otorga también el valor verdadero a la conclusión 5., pues esta no puede ser falsa "a menos que concedamos que l. es falsa, en cuyo caso estaríamos incurriendo en contradicción. Por tanto, el esquema de demostración propuesto, tomado en su conjunto, si garantiza la construcción de una inferencia lógicamente válida y el establecimiento de una regla de deducción lógicamente conclusiva para legitimar la separación del consecuente de l. de su respectivo antecedente. Por consiguiente, 0 bien se asume la validez del esquema de prueba propuesto y con ello que el MP es una regla de inferencia en toda prueba por inducción matemática, o bien se sostiene lo contrario y se concede que la conclusión de la línea 5. es gratuita con lo que se estaría aceptando que realmente no

(7) Anotamos que el ejemplo de la estructura matemática no indiciciva basta para probar que el PIM no es una proposición lógicamente verdadera $y$, consecuentemente, carece de la universalidad necesaria que usualmente se reclama para los juicios sintéticos a priori, lo cual puede entenderse como una limitación para las pretensiones de Poincaré pero al mismo tiempo para las posiciones logicistas derivadas de Russell. 
hay pruebas inductivas y con ello un absurdo desde el punto de vista matemático y una limitación lógica que no existe (8).

Este resultado nos permite sostener que el MP es condición necesaria para la aplicación del PIM y que éste, a su vez, es condición suficiente para asegurar que el MP ha sido utilizado. Asimismo, en la medida que las condiciones necesarias de una formulación no son otra cosa que los presupuestos para su validez, vale decir, su lógica subyacente, luego podemos aseverar que la llamada regla de separación forma parte de la lógica subyacente en toda demostración por inducción matemática.

2. Pueden hacerse dos observaciones sustantivas al plantea. miento antes descrrollado. La primera sería el señalar que es una cuestión de hecho que se han construido sistemas lógicos satisface torios, como el M.J. Nicod, que utilizan una regla de inferencia distinta a la aquí postulada,y, consecuentemente, podría sostenerse que si se usara dicho sistema para la formalización de la aritmética, no sería necesario presuponer al MP. La segunda podría ser tomada de una de las consecuencias más interesantes del llamado teorema fundamental de Herbarand que implica que el MP es omitible en la teoría de la cuantíficación, la que puede construirse utilizando las denominadas "reglas del pasaje", las dos reglas de generalización y la regla generalizada de simplificación. La corrección de estos resultados, con sus respectivas limitaciones, ha sido verificada y comentada, entre otros, por Burton Dreben en sus notas adjuntas a la publicación de los trabajos de Herbrand contenidos en From Frege to Gödel de van Heijenoort.

Nos dedicaremos ahora a observar con detalle la primera observación. Nicod (9) construyó un sistema lógico reduciendo los usuales operadores de la lógica proposicional a solamente el operador de incompatibilidad con el cual es posible construir un sistema funcionalmente completo debido a que permite expresar cualquiera de los 16 operadores binarios posibles. Dadas las restricciones del lenguaje de Nicod, no es posible formular en su sistema directamente del MP sino su equivalente. Sin embargo la re.

(8) In el sistema de Peano contenido en Los principios de la aritmética presentados por un nuevo método, el MP no aparece como regla de inferencia lo que da lugar a que las pruebas inductivas, desde el punto de vista estrictamente formal sean gratuitas. Pero esta insuficiencia no debe entenderse en el sentido de que Peano sostuvo que su axiomatización no necesitaba del MP sino que no lo hizo explicito, lo cual fue una deficiencia formal $y$ es compatible con nuestro planteamiento.

(9) Fi sistema de Nicod puede verse en Copi, Symbolic Logic, 3a. ed., p. 208. 
gla de inferencia usada por Nicod no es exactamente equivalente a la forma canónica del MP, pues su esquema es el siguiente:

"Desde P y P. / . R / Q se infiere Q"

Realizando conocidas transformaciones, encontramos que el equivalente en notación usual de la regla de Nicod es:

"Desde P y $P \rightarrow(R . Q)$ se deduce $Q "$ ".

Como puede verse fácilmente, el MP es un caso particular de este esquema cuando se sustituye $R$ por $Q$ (10). Pero, además, se puede constatar sin dificultad que la regla de Nicod equivale a la aplicación sucesiva del MP y de la regla de simplificación, lo que prueba que la tradicional regla de inferencia es condición necesaria para la deducción en el sistema examinado.

Para examinar la posible objeción basada en una de las consecuencias del llamado teorema fundamental de Herbrand, contenido en su trabajo Investigations in proof theory (1930), es necesario traer a colación algunas características de su sistema, que es usualmente denominado con la abreviatura $Q_{\mathrm{H}}$. En esta exposición prescindiremos de detalles técnicos muy elaborados y nos limitaremos a proporcionar, informalmente pero con precisión, aquello que es necesario para entender el sentido del argumento.

(i) Las llamadas "reglas del pasaje", que pueden denotarse por el par $J_{h} ; K_{h}$, para $h=1, \ldots, 6$, son:

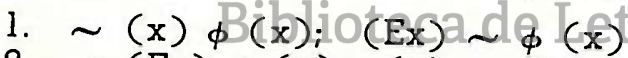

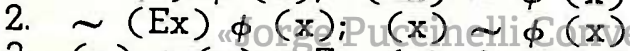

3. $(x) \phi(x) \vee Z ;(x)(\phi(x) \vee Z)$

4. Z v (x) $\phi(x)_{i}(\mathrm{x})(\mathrm{Z} \mathrm{v} \phi(\mathrm{x})$ )

5. $(E x) \phi(x) \vee Z_{;}(E x)(\phi(x) \vee Z)$

6. $\mathrm{Z} \vee(E x) \phi(x) ;(E x)(Z \vee \phi(x))$

En estos pares de fórmulas se asume que la variable " $x$ " no es libre en Z. Conmutando las fórmulas componentes obtenemos el par $K_{h} ; J_{h}$, con lo que totalizamos 12 "reglas del pasaje" para el sistema del Herbrand. Asimismo es un resultado conocido, expresado en $\mathrm{Q}_{\mathrm{H}}$ por la proposición 3.101, que para cualquier proposición $\mathrm{P}$ se puede obtener otro equivalente $\mathrm{P}^{\prime}$ por aplicación de las reglas anteriores. La fórmula $\mathrm{P}^{\prime}$ se denomina forma normal prenex $\mathrm{y}$ es de la forma:

$$
\left(Q_{1} Y_{1}\right) \ldots\left(Q_{n} Y_{n}\right) \phi\left(Y_{1} \ldots . Y_{n}\right)
$$

(10) Este resultado fue señalado por L. Post en Introduction to a general theory of elementary propositions, cifra 7 . 
en la que cada $\left(Q_{i} y_{i}\right)$ es un cuantificador universal o existencial que liga a alguna de las variables $y_{i}$, las cuales son todas distintas entre sí. Cuando todos los cuantificadores existenciales anteceden a todos los cuantificadores universales, la fórmula es conocida como forma normal de Skolem.

Una fórmula $P$ es una identidad normal si existe alguna forma normal prenex $P^{\prime}$ de ella, desde la cual es derivable por medios conocidos una identidad del tipo

$$
\phi(x) v \sim \phi(x)
$$

que Herbrand denomina identidad de primera clase.

Una proposictón $\mathrm{P}$ tiene la propiedad $A$ si es posible derivar desde ella, mediante un proceso de sustitución de esquemas de cuantificadores (11), una proposición $P^{\prime}$ que es una identidad normal.

Si una proposición $\mathrm{P}$ tiene la propiedad $A$, entonces existe en el sistema $Q_{\mathrm{H}}$ una prueba para $P(12)$. En breve, si $P$ tiene la propiedad A,entonces $\mid-A$. Recíprocamente, si $P$ es demostrable Q en el sistema $Q_{\mathbf{B}}$, entonces $\mathrm{P}$ tiene lar propiedad $A$.

Herbrand establece como resultado a partir de su teorema fundamental (13) que la demostración en $Q_{H}$ de una proposición $P$. que satisface la propiedad $A$, precisa única y exclusivamente de la aplicación de las "reglas del pasaje", de las reglas de generalización y de la regla generalizada de simplificación.

Conocida la forma lógica de las "reglas del pasaje" y de las reglas de generalización universal y existencial, es fácil constatar que ninguna de ellas legitima la operación de separación. Esto es, no nos permiten afirmar a partir de una proposición dada la verdad de una de sus proposiciones componentes, por lo que no hay razón para que alguna de ellas presuponga el MP. Por tonto, de las reglas necesarias para construir una demostración en $\mathrm{Q}_{\mathrm{H}}$, solamente la regla generalizada de simplificación podría pre-

(11) Un "esquema de cuantificadores" y un "tipo" son nociones técnicas en el texto de Herbrand que seria laborioso e innecesario considerar aquí en detalle. Asimismo no existe un algoritmo para decidir, en general, si cualquier proposición $P$ tiene la propiedad A.

(12) Este es un resultado de Herbrand que establece que una teoria de primer orden es semánticamente completa, teorema cuya prueba se atribuye usualmente solo a Gödel (1930). Como lo señala Wang en Eigthy years of foundational studies, Herbrand no concedió especial importancia a su demostración debido a que la noción de compleción no era significativa para él debido a que aceptaba solamente métodos finitisticos.

(13) Vid. van Heijenoort, From Frege to Gödel, Cambridge, Harvard University Press, 1967, p. 558. 
suponer la validez del MP, pues, como veremos a continuación al formular su enunciado, ésta si permite la separación de uno de sus componentes.

Regla generalizada de simplificación Df. "Si en una proposición verdadera (demostrable en $Q_{H}$ ) la subproposición $P$ V P es reemplazada por $P$, entonces obtenemos otra proposición verdadera" (14).

La validez de esta regla derivada en $Q_{H}$ supone la demostrabilidad de la fórmula $\mathrm{P} \vee \mathrm{P} \equiv \mathrm{P}$ dentro de dicho sistema. En efecto, como Burton Dreben lo ha señalado en detalle en su nota $B$ a la edición consultada, este teorema se establece demostrando en $\mathrm{Q}_{\mathrm{K}}$ "P $\mathrm{P} \mathrm{P} \rightarrow \mathrm{P}$ " $\mathrm{y}$ "P $\rightarrow \mathrm{P} \vee \mathrm{P}^{\prime \prime}$ sin necesidad de recurrir a la " $\mathrm{re}$ gla de implicación" (15). Por tanto, la prueba en $Q_{\mathrm{B}}$ de una proposición $\mathrm{P}$, que tiene la propiedad $A$, puede realizarse sin ninguna dificultad con independencia del MP usando la regla generalizada de simplificación para garantizar el rigor de la operación de separación. Este resultado se sigue claramente de la demostración antes descrita $y$ es considerada por Herbrand de la mayor importancia. Dreben por su parte opina que es el resultado básico de la tesis sostenida en Investigations in proof theory.

Sin embargo, la demostración reseñada no tiene la fuerza suficiente como para sustentar una afirmación en el sentido de que la aritmética sea formalizable sólo con los medios expresivos de $Q_{\mathrm{H}}$ de tal manera que no sea necesaria la "regla de implicación". Esta posibilidad, que debilitaría significativamente nuestro planteamiento, realmente tolo existe, en da medida que el mismo Herbrand anotó como una limitación de las consecuencias de su teorema fundamental que el MP no es omitible en "una teoría matemática que contenga hipótesis. Nuestro autor no entra en mayores detalles para fundamentar la exactitud de este último aserto, pero la argumentación que dimos para probar que la mencionada regla es condición necesaria para el uso del PIM, es suficiente para avalar la aserción herbrantiana con la única condición que el referido principio sea una hipótesis.

Ciertamente no es necesaria mayor argumentación para probar que el PIM es una hipótesis. Al respecto basta señalar que es una proposición necesaria para derivar específicamente las proposiciones verdaderas de la aritmética y por ello hay que añadirla en las formalizaciones de ella a los enunciados estrictamente lógicos, como lo hace Herbrand tanto en Investigations in proof

(14) Vid. ídem.

(15) Detalles de esta demostración pueden ser consultados en las notas A, B y D hechas por Berton Dreben a los textos de Herbrand contenidos en From Frege to Gödel. 
theory como en su trabajo On the consistency of arithmetic (1931) (16).

3. Finalmente, como una limitación adjetiva de la argumentación precedente podría, tal vez, observarse que nuestro planteamiento carece de generalidad por que no afecta a la versión fuerte del PIM. Sin embargo, sin excluir otras posibles respuestas a esta objeción, nos limitaremos a puntualizar que nuestro planteamiento si es válido para la denominada versión fuerte del principio analizado, en la medida que ésta puede ser demostrada a partir de la enunciación débil. Al respecto pueden encontrarse pruebas alternativas con relativa facilidad, pero la que nosotros brindaremos a continuación tiene la ventaja, para nuestros fines, de haber sido construida dentro de una teoría formalizada de números en la que se hace explícita la lógica subyacente.

Antes de proseguir, también es oportuno advertir que hemos optado por una formalización del PIM que podría no ser aceptada por ciertas posiciones intuicionistas. Esto es, lo expresamos a través de una fórmula lógica que contiene variables ligadas por cuantificadores, que son denominadas, dentro de una tradición iniciada por Whitehead y Russell, variables dparentes. Esta formalización tiene importantes diferencias teóricas con la presentada, por ejemplo, por Herbrand en su demostración de la consistencia de la aritmética, la cual no contiene variables aparentes. De otra parte, anotamos que es generalmente a la versión fuerte a la que se denomina Principio de Inducción Completa.

La fórmula que demostraremos cómo teorema es la siguiente:

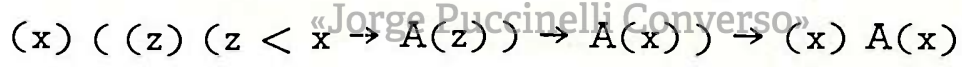

En la demostración se utilizará el teorema de la deducción, y se tomarán como premisas algunos teoremas de la Teoría Formal de Números de Mendelson (17), los que listaremos a continuación. También se utilizarán como reglas de inferencia algunas tautologías, que por ser muy conocidas no hace falta que las especifiquemos previamente, y las reglas de introducción y eliminación de cuantificadores.

Tl. $\sim(\mathrm{t}<0)$

T2. $A(0) \cdot A(1) \ldots . . A(k) \equiv(x)(x \leqslant k \rightarrow A(x))$

T3. $\mathrm{t} \leqslant \mathrm{r} \equiv \mathrm{t}<\mathrm{r}^{\prime}$ (En adelante para designar al sucesivo de $\mathrm{t}$ usaremos la expresión $t^{\prime}$ )

(16) Contenido en From Frege to Gödel, pp. 618-628.

(17) Vid. E. Mendelson. Introduction to Mathematical Logic. Toronto, D. Van Nostrand Company, Inc., 1966, cap. III. 
T4. $x=y \rightarrow(A(x, x) \rightarrow A(x, y))$

D2. $z \leqslant x^{\prime} \equiv z<x^{\prime} v z=x^{\prime}$

T5. $\mathrm{x} \leqslant \mathrm{x}$

La demostración es como sigue:

I. Supongamos una propiedad tal como $B(x)$ que sea en detalle de la forma $(z)(z \leqslant x \rightarrow A(z))$. Probaremos primero en esta primera parte que 0 tiene la propiedad $B$, esto es $B(0)$. En la segunda parte probaremos que es verdad $B\left(x^{\prime}\right)$ siempre que se suponga que se cumple la hipótesis inductiva $B(x)$.

1. $(x)((z)(z<x \rightarrow A(z)) \rightarrow A(x))$ Premisa.

2. $(z)(z<0 \rightarrow A(z)) \rightarrow A(0)$ EU. en 1 .

3. $\sim(z<0)$

4. $(z<0 \rightarrow \bar{A}(z))$

Tl.

5. (z) $(z<0 \rightarrow A(z))$

$\mathrm{Tau} \sim \mathrm{p} \rightarrow(\mathrm{p} \rightarrow \mathrm{q}) \mathrm{y}$

6. $A(0)$

7. $(z)(z \leqslant 0 \rightarrow A(z)$

MP. con 3.

8. $B(0)$ 3, GU.

9. $(x)((z)(z<x \rightarrow A(z)) \rightarrow A(x)) \vdash B(0)$; Hay

2 y $5 \mathrm{MP}$.

6. T2. (para $k=0$ )

7 es $B(0)$ una prueba de que $B(x)$

se cumple para $x=0$ )

1. $(x)(z)(z<A(z)) \rightarrow A(x))$ Premisa.

2. (z) $(z \leqslant x \rightarrow A)(z))$

3. (z) $\left(z<x^{\prime} \rightarrow A(z)\right)$

Hipótesis inductiva $B(x)$

4. (z) $\left(z<x^{\prime} \rightarrow A(z)\right) \rightarrow A\left(x^{\prime}\right)$ asl, EU.

5. $A\left(x^{\prime}\right)$

$3,4 \mathrm{MP}$.

6. $z \leqslant x^{\prime} \rightarrow z^{r}<x^{\text {Puccinelli Converso }} \mathbf{z}=\mathrm{x}^{\text {D. }} 2$.

7. $z<x^{\prime} \rightarrow A(z)$

8. $z=x^{\prime} \rightarrow\left(A\left(x^{\prime}\right) \rightarrow A(z)\right)$

9. $A\left(x^{\prime}\right) \rightarrow\left(z=x^{\prime} \rightarrow A(z)\right)$

3, EU.

T4. Simetría

En 8 Exportación, Conmut. y nuevamente Ex-

10. $z=x^{\prime} \rightarrow \bar{A}(z)$ port.

11. $z \leqslant x^{\prime} \rightarrow A(z)$

5,9 MP.

$6,7,10$, por tautología $((\mathrm{p} \rightarrow(\mathrm{q} v \mathrm{r}))$. $(q \rightarrow z) \cdot(r \rightarrow z)) \rightarrow$ $(p \rightarrow z)$

12. $(z)\left(z \leqslant x^{\prime} \rightarrow A(z)\right)$

Gen. en 11 .

13. $B\left(x^{\prime}\right)$ 12 es $B\left(x^{\prime}\right)$

14. $(x)((z)(z<x \rightarrow A(z)) \rightarrow A(x)) \vdash(x)(B(x) \rightarrow$ $\left.B\left(x^{\prime}\right)\right)$

Este paso se justifica por aplicación del Teorema de la Deducción y Generalización U. 
III. Desde lo establecido en I y II y el Principio de Inducción Matemática obtenemos $\mathrm{P} \vdash(\mathrm{x}) \mathrm{B}(\mathrm{x})$. Vale decir, hemos obtenido una prueba a partir de la premisa P (P designa abreviadamente la primera línea de I y la primera línea de II) que nos permite generalizar la propiedad $B(x)$ a todo número natural. Ahora, por aplicación reiterada de la regla de Ejemplificación Universal obtenemos $P \vdash x \leqslant x \rightarrow A(x), y$ por T5. y MP. tenemos $P \vdash A(x), y$, aplicando la regla de Generalización Universal y nuevamente el Teorema de la Deducción, $\vdash P \rightarrow(x) A(x)$ que, detallando $P$, es $\vdash(x)$ $((z)(z<x \rightarrow A(z)) \rightarrow A(x)) \rightarrow(x) A(x)$.

Antes de terminar este artículo hacemos la salvedad de que aunque nuestra formalización del Principio de Inducción Matemática no es necesariamente compatible con el intuicionismo, sin embargo, nuestra tesis sobre el estatuto de condición necesaria atribuido a la "regla de implicación", es compatible con la llamada lógica intuicionista de Gentzen (18) que la incluye como una de sus reglas primitivas de inferencia. De igual manera, Andrei $\mathrm{Ni}$ kolaevich Kolgomorov en su artículo, On the principle of excluded middle (1925) desarrolla una lógica intuicionista, aprovechando los resultados Brouwer, en términos algo más exigentes que los que posteriormente utilizara Heyting. Kolgomorov prueba que el sistema de Hilbert (1922) para la lógica proposicional es reducible a su sistema B que permite desarrollar la matemática sin la ayuda del principio del tercio excluido. Este sistema intuicionista también es compatible donOnuestro Cplanteámiento en la medida que utiliza el MP comoreggla de separaciónerso"

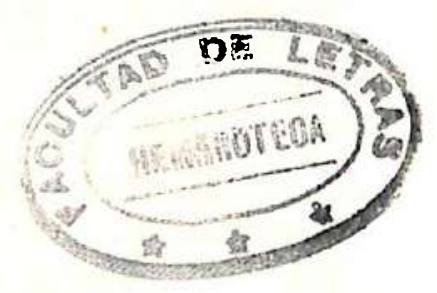

(18) Nos referimos al sistema conocido como $\mathrm{NJ}$, aunque nuestra tesis se cumple también para NK. 